\title{
27
}

\section{Implementation of Positive Education Projects in Hong Kong}

\author{
Sylvia Kwok
}

Positive education, in part, refers to the application of positive psychology concepts to education settings so as to foster positive wellbeing and adaptive functioning (Norrish, Williams, O'Connor, \& Robinson, 2013; Seligman, 2012). Over the past decade, various activities and approaches have been developed to enhance the wellbeing of students, teachers, and parents in schools. Character strengths are foundational in positive psychology, and wellbeing has been delineated in the PERMA model (Seligman, 2012) as: positive Emotions (to build positive emotional experiences and cope with negative emotions), positive Engagement (to live a life high in interest, curiosity, and absorption), positive Relationships (to develop social and emotional skills and foster nourishing relationships with self and others), positive Meaning (to engage in activities that serve a greater cause and act in accordance with individual values), and positive Accomplishment (to develop confidence and competence through striving for and achieving meaningful outcomes). These five domains supplement and interact with one another, supporting people to build a happy and flourishing life, and, as such, preventing them from developing mental health problems (Seligman, 2012).

Character strengths are the psychological processes or mechanisms that define a set of virtues, which are the core and universal characteristics valued by moral philosophers (Peterson \& Seligman, 2004). The Values in Action framework (Peterson \& Seligman, 2004), suggests that there are 6

\section{S. Kwok (凶)}

City University of Hong Kong, Kowloon Tong, Hong Kong e-mail:scyckwok@cityu.edu.hk 
virtues, together comprised of 24 character strengths, and provides a helpful approach to character strengths within positive education. The six virtues are: wisdom and knowledge, courage, humanity, justice, temperance, and transcendence. Wisdom and knowledge are cognitive strengths that include creativity, curiosity, love of learning, judgement, and perspective. Courage involves emotional strengths incorporating bravery, persistence, integrity, and zest. Humanity refers to interpersonal strengths that comprises love, kindness, and social intelligence. Justice refers to civic strengths that consist of teamwork, fairness, and leadership. Temperance involves strengths that protect us against excess, such as forgiveness, humility, prudence, and self-regulation. Transcendence is comprised of strengths that connect us to the larger universe and provide meaning in our lives, and includes appreciation of beauty and excellence, gratitude, hope, humour, and spirituality.

In view of the need for preventive and developmental programs to promote the positive mental health of people, in 2012 we established a positive education laboratory in the Department of Social and Behavioural Sciences at the City University of Hong Kong. Throughout these years, we have been launching a number of positive education projects for the kindergarten, primary, and secondary (high) schools, as well as universities in Hong Kong.

\section{Six Level Implementation Process}

The positive education projects in the Hong Kong schools are guided by a six-level implementation process described as learn it, live it, reflect it, conceptualize it, apply it, and embed it. This process was developed by integrating experiential learning theory (Kolb, Boyatzis, \& Mainemelis, 2001) and the applied model of positive education in the Geelong Grammar School in Australia (Norrish et al., 2013). Learn it refers to regular learning opportunities provided to students, teachers, and parents to understand the science of wellbeing. Live it encourages participants to enact evidence-based wellbeing practices in their unique ways, both in schools and in their daily lives. Reflect it and conceptualize it means that individuals are assisted to reflect on what they have learned and experienced so as to conceptualize their experiences with a deeper understanding of the concepts and principles of positive education. The process of apply it implies the actual application of designing and conducting positive education programs or activities in schools and communities. Finally, embed it advocates building long-term, school-wide policies, and a positive culture which support and nurture wellbeing within individuals, schools, and the community. The embed it component captures the 
macro level initiatives in a school, and, in combination with other processes, represents a critical process for successful and sustainable implementation (Hoare, Bott, \& Robinson, 2017).

This six-level implementation process serves as a guide for delivering positive education and can be repeated in cycles. It is not just a pedagogy, but a way of life within the school and in daily activities. More importantly, when designing and implementing the interventions with schools, the unique needs of a school will be assessed to ensure that the initiatives are tailored to be context-specific to individual schools. We leverage existing practice and build on a school's unique strengths, while providing opportunities for individual schools to activate changes in the school system.

\section{Pioneering Positive Education Projects in Pre-primary Schools}

Focusing on the character strengths of creativity, bravery, hope, love, altruism, honesty, gratitude, and forgiveness, a one-year positive education project was launched in five nurseries and three kindergartens. A whole school positive education approach was adopted. Teacher training on positive education and parents' workshops on positive parenting were conducted. The schools also adopted the above-mentioned themes in their monthly programs (e.g., birthday parties, celebration of Mid-autumn Festival, Ching Ming Festival, Christmas, Chinese New Year, Mothers' Day, and Fathers' Day). A positive education curriculum was designed for 368 Kindergarten K2 and K3 students aged 4-6 (187 in the experimental group, 181 in the control group). Two lessons, 45 minutes per lesson, were designed on each theme, resulting in a total of 16 positive education lessons. The lessons were taught by a psychologist with training and experience in early childhood education and positive education programs, with assistance from the teachers. There were about 15 students in each group. A wait-list randomized controlled trial research design was adopted. The students were randomized into experimental and control groups, with the commitment that the lessons would be offered to children in the control group after the study ended. Parents completed the same set of questionnaires on their children before and after the intervention. The questionnaires consisted of measures such as an anxiety and depression scale, a children's hope scale, a gratitude questionnaire, an altruism scale, a forgiveness scale, an honesty attitudes scale, and a courage measure.

Results showed that students in the experimental group increased significantly in forgiveness, while decreasing significantly in anxiety, when compared with the control group. This suggests that positive education 
is effective in enhancing positive attributes and decreasing mental health problems in pre-primary school students.

\section{Pioneering Whole School Positive Education Project in Primary Schools}

Whole school positive education was launched in six primary schools in a two-year period to enhance the wellbeing of students and teachers. The strengths, weaknesses, opportunities, and threats of the schools were analysed, while recommendations on positive education development were made to the schools. The school boards were contacted, and school management teams all showed support for the implementation of the project. Principals formed core positive education teams in the schools, comprised of guidance teachers, counsellors, and social workers so as to facilitate implementation. Teacher training and parent workshops were conducted, while positive education elements were integrated within student activities, such as in school assemblies, sporting events, swimming events, leadership camp, study tours, school carnivals, and so forth. Special parent-child activities were implemented, such as candle-night dinner and joint video games to promote parent-child relationship. The positive education curriculum was designed for 500 Grade 1 to Grade 6 students aged 6-13 with the PERMA themes. A total of 16 lessons were designed for each grade, with each lesson lasting for about 45 minutes. A quasi-experimental research design was adopted with pre- and post-intervention questionnaires completed by the students, teachers, and parents. The questionnaires consist of measures such as the PERMA Profiler (Butler \& Kern, 2016), an academic motivation scale, a parent-child conflict tactics scale, and a hospital anxiety and depression scale.

Results showed that positive education was effective in decreasing students' negative emotions and anxiety, increasing their motivation in completing tasks, developing their positive personality, and alleviating perceived inappropriate parenting methods such as parental abuse and neglect. For parents, positive education was effective in enhancing positive emotions, engagement, growth mindset and alleviating communication problems and family difficulties. Positive education also helped to improve teacher-student relationship and constructive teaching for teachers. Hence, whole school positive education was effective at enhancing wellbeing and decreasing psychopathology for primary school students; promoting the growth mindset of parents and decreasing parent-child communication problems; and improving teacherstudent relationships. 


\section{Pioneering Positive Education Projects in High Schools}

Focusing on optimism, hope, and character strengths, the positive education project was launched in ten high schools for a two-year period to help decrease the anxiety of students. A six-session positive education curriculum was designed by a team of social workers and educational psychologists with reference to the above-mentioned themes for 1,290 Grade 10 to Grade 12 students aged 14-18. Each lesson lasted for one hour and was taught by a social worker in class. There were 30 students in each class. A waitlist randomized controlled trial research design was adopted. Two classes of students in one grade were randomly selected as the experimental group, while the remaining two classes acted as the control group. There was a total of 670 students in the experimental group, and 620 students in the control group. The students in the control groups were provided with the positive education lessons after the study ended. All students completed the same set of questionnaires before and after the intervention. The questionnaire consisted of measures such as the hospital anxiety and depression scale, a subjective happiness scale, a children's hope scale, and an optimism scale. Results showed that students in the experimental groups had significant increases in subjective happiness, hope, and life orientation, and significant decreases in anxiety after the intervention. This suggests that positive education is effective in increasing positive attributes, such as optimism, hope, and subjective happiness, while decreasing mental health problem, such as anxiety, in high school students.

\section{Pioneering Positive Education Projects in Universities}

A joint university positive education project was launched in five universities in Hong Kong for three years. The project aimed to nurture and enhance the development of students' positive emotions, relationship, purpose, accomplishment, engagement and health, and to increase students' life satisfaction, strengthen their resilience in the face of difficulties, and enhance understanding and support teaching of positive education for academic staff at the tertiary level.

To facilitate acquisition of knowledge in positive education, a number of training camps, workshops, and seminars were organized for students and staff at the universities. Students were encouraged to write gratitude journals and gratitude letters, record their memorable moments in the book of blessing, practice mindfulness at home, and set their own goals and devise strategies to achieve the goals so as to live out the concepts in their daily lives. 
They were required to write reflective journals or reflective notes in a webbased platform, have regular meetings with and presentations to the project officers to share and reflect what they have learned. They were encouraged to present their learning and reflections at the International Conference on Positive Education, hence reflection and conceptualization of positive education were attained. To apply the positive education concepts, students were divided into small groups and launched different community projects under the supervision of the project officers. They also organized positive education festivals at their own universities and held a study tour to Taiwan to learn and share their experiences. To embed the positive education elements into their universities, they set up what went well boards; distributed pamphlets, cards with mottos, and gave souvenirs to other students; organized exhibitions; and established a positive education website to disseminate positive education messages to students and the public.

Surveys were administered before and after students' participation in the activities. Different measures were used to examine the change in students' emotions, relationships, meaning of life, accomplishments, engagement, and resilience. Results indicated that students had significant increases in college life satisfaction, relationship satisfaction, adaptive coping, and decreases in anxiety and stress arising from time pressures. It appears that positive education was effective in enhancing the wellbeing and decreasing mental health problems of university students.

\section{Characteristics of the Positive Education Projects}

Several characteristics can be identified from the above-described projects. Applying a positive education and experiential learning framework, the projects aimed to enhance the wellbeing of students, teachers, and parents; to prevent mental health problems; and to promote a positive culture in the schools. The project objectives are concrete, measurable, and achievable, with the development of localized positive education curricula that incorporate Chinese cultural and moral values for students of different age groups. The teaching pedagogy was comprised of six steps that included Learn, Live, Reflect, Conceptualize, Apply, and Embed. A whole school approach to positive education was adopted in the schools, rather than fragmented group or program interventions. Involvement of school management personnel (e.g., school board members, school administrators), parents, and teachers was emphasized. Under the positive education and experiential learning framework, some additional programs were tailor-made for individual schools. 
The framework was integrated with the mission and vision of the individual schools and integrated into the school development plan and school policy. The lessons or programs were designed with multiple formats, including experiential exercises, mindfulness activities, games, role play, video show, drama, arts, and music with detailed debriefing that met the needs and characteristics of the students, teachers, and parents.

In addition, the projects were implemented by a multi-disciplinary team comprised of academics, social workers, psychologists, ex-teachers, and research fellows with doctorates in social work, psychology, and education. The projects had both research and knowledge transfer elements. The participating schools were encouraged to share their experiences with other schools, creating a ripple effect while sustainability is maintained. Joint advocacy with the funding bodies was made such that positive education messages could be promoted to other professionals, schools, parents, the media, and the public.

\section{Factors Affecting Effectiveness of the Positive Education Projects}

The effectiveness of the whole school positive education approach is influenced by school, teacher, classroom, and student factors. The underlying mechanism impacting effectiveness of the whole school positive education approach is impacted by an array of variables. Teachers' implementation quality can be affected by the school atmosphere, their social-emotional competence, their attitudes towards positive education, their training, and the consultation they received. A flexible school policy, placing less emphasis on students' academic performances and more on students' wellbeing, will facilitate the implementation of positive education. A harmonious relationship among students, teachers, and parents is also a facilitating factor for effective implementation. In addition, teachers' implementation quality may interact with classroom climate, which in turn impacts students' wellbeing. At the same time, students' wellbeing may be affected by their engagement in class, as well as their perceived parenting and friendship quality.

\section{Future Directions for Positive Education in Hong Kong}

The emphasis for future implementation of positive education in Hong Kong (and elsewhere) includes: 
1. Positive education should be embedded in formal/informal school curriculum and school activities.

2. The themes of positive education should be integrated into school development plans and policies.

3. More evidence-based research is needed to systematically examine the conceptual framework and implementation approaches of positive education and to provide evidence of the effectiveness of positive education for individual and collective wellbeing in schools and the communities.

4. Multiple teaching pedagogies should be developed for the explicit and implicit teaching of positive education (e.g., mindfulness training, expressive arts, music, art, drama, movement, etc.) that can be incorporated in the positive education curriculum.

5. Positive education should be extended to different target groups (e.g., students with special education needs).

6. Continuous discovery and innovation in positive teaching and learning, positive training, positive research, positive organization, positive resources (including online materials), positive knowledge development should be promoted.

7. Students, teachers, and parents should all be involved in the design and implementation of positive education.

8. Collaboration among local and international academics and educators in promoting positive education should be encouraged.

9. An interdisciplinary platform should be established to enhance collaboration among schools, social welfare agencies, business sectors, government, and universities in promoting positive education.

\section{References}

Butler, J., \& Kern, M. L. (2016). The PERMA-Profiler: A brief multidimensional measure of flourishing. International Journal of Wellbeing, 6, 1-48. https://doi. org/10.5502/ijw.v6i3.526.

Hoare, E., Bott, D., \& Robinson, J. (2017). Learn it, live it, teach it, embed it: Implementing a whole school approach to foster positive mental health and wellbeing through positive education. International Journal of Wellbeing, 7(3).

Kolb, D. A., Boyatzis, R. E., \& Mainemelis, C. (2001). Experiential learning theory: Previous research and new directions. Perspectives on Thinking, Learning, and Cognitive Styles, 1(8), 227-247. 
Norrish, J. M., Williams, P., O’Connor, M., \& Robinson, J. (2013). An applied framework for positive education. International Journal of Wellbeing, 3(2).

Peterson, C., \& Seligman, M. E. (2004). Character strengths and virtues. New York, NY: Oxford University Press.

Seligman, M. E. (2012). Flourish: A visionary new understanding of happiness and well-being. New York, NY: The Free Press.

Open Access This chapter is licensed under the terms of the Creative Commons Attribution 4.0 International License (http://creativecommons.org/licenses/by/4.0/), which permits use, sharing, adaptation, distribution and reproduction in any medium or format, as long as you give appropriate credit to the original author(s) and the source, provide a link to the Creative Commons license and indicate if changes were made.

The images or other third party material in this chapter are included in the chapter's Creative Commons license, unless indicated otherwise in a credit line to the material. If material is not included in the chapter's Creative Commons license and your intended use is not permitted by statutory regulation or exceeds the permitted use, you will need to obtain permission directly from the copyright holder.

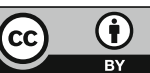

